\title{
FIRST ORDER CALCULI WITH VALUES IN RIGHT-UNIVERSAL BIMODULES
}

\author{
ANDRZEJ BOROWIEC \\ Institute of Theoretical Physics, University of Wroctaw \\ Pl. Maxa Borna 9, 50-204 Wroctaw, Poland \\ E-mail: borow@ift.uni.wroc.pl \\ VLADISLAV KHARCHENKO \\ Centro de Investigaciones Teoricas, FESC, UNAM \\ Apartado Postal 95, C.P. 54700 Cuautitlán Izcalli, Estado de México \\ E-mail: vlad@servidor.unam.mx \\ ZBIGNIEW OZIEWICZ \\ Facultad de Estudios Superiores, Cuautitlán \\ Universidad Nacional Autonoma de México \\ Apartado Postal 25, C.P. 54700 Cuautitlán Izcalli, Estado de México \\ E-mail: oziewicz@servidor.unam.mx
}

\begin{abstract}
The purpose of this note is to show how calculi on unital associative algebra with universal right bimodule generalize previously studied constructions by Pusz and Woronowicz [1989] and by Wess and Zumino [1990] and that in this language results are in a natural context, are easier to describe and handle. As a by-product we obtain intrinsic, coordinate-free and basisindependent generalization of the first order noncommutative differential calculi with partial derivatives.

1. Introduction. In this note $\mathbb{l}$ denotes some fixed unital and commutative ring. Algebras are unital associative $\mathbb{k}$-algebras and homomorpisms are assumed to be unital. The tensor product $\otimes$ means $\otimes_{\mathbb{k}}$ and linear map means $\mathbb{k}$-linear. All objects considered here are first of all $k$-modules. All maps are assumed to be $k$-linear maps. Most results in

1991 Mathematics Subject Classification: Primary 16D20; Secondary 16W25, 16 U80.

Research partially supported by KBN grant 2 P302 02307.

The second author wishes to thank CONASyT-Mexico for its support, Catedra Patrimonial, 940411 and also the Russian Foundation for Fundamental Research, grant 95-01-01356.

Z. Oziewicz is a member of Sistema Nacional de Investigadores, México.

The paper is in final form and no version of it will be published elsewhere.
\end{abstract}


Section 3 will be set under additional assumption that $l k$-modules $V$ and $W$ are projective.

1.1. First order differential calculus as a derivation of an algebra. Let $A$ be an algebra with a multiplication $m \in \operatorname{lin}\left(A^{\otimes 2}, A\right), M$ be an $(A, A)$-bimodule ( $A$-bimodule in short).

It appears that differential calculi investigated recently by many authors in the context of quantum groups and noncommutative geometry are nothing but dervations of an algebra with values in a bimodule. Recall that a $\mathbb{k}$-derivation $d$ of $A$ to $M, d \in \operatorname{der}_{\mathbb{k}}(A, M)$, is a $l k$-linear mapping from $A$ into $M$ such that the Leibniz rule

$$
d(x y)=d x . y+x \cdot d y
$$

holds true for each $x, y \in A$ where, dot "." denotes both (left and right ) multiplications by elements from $A$.

Definition 1. The triple $\{A, M, d\}$ is said to be first order calculus or first order differential on an algebra $A$ with values in an $A$-bimodule $M$ or shortly $M$-valued calculus on $A$.

Each $k$-derivation vanishes on scalars from $\mathbb{k}$.

1.2. Functorial properties. The following functorial properties of derivations are well known [2]:

Proposition 1.2.1. (Bourbaki 1989, $\$ 10.7$, Prop. 9). Let $A, B$ be two algebras, $M$ an A-bimodule and $N$ a B-bimodule; let $\phi: A \longrightarrow B$ be an algebra homomorphism and $\Phi: M \longrightarrow N$ an $A$-homomorphism of $A$-bimodules (relative to $\phi$ ). Then

(i) For every $d_{B} \in \operatorname{der}_{\mathbb{k}}(B, N), d_{B} \circ \phi \in \operatorname{der}_{\mathbb{k}}\left(A, \phi^{*}(N)\right)$.

(ii) For every $d_{A} \in \operatorname{der}_{\mathbb{k}}(A, M), \Phi \circ d_{A} \in \operatorname{der}_{\mathbb{k}}\left(A, \phi^{*}(N)\right)$, where $\phi^{*}(N)=N$ with an $A$-bimodule structure induced by $\phi$.

We are interested in the case when the diagram

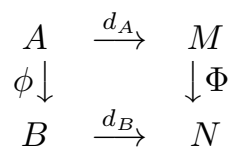

commute. The condition of commutativity

$$
\Phi \circ d_{A}=d_{B} \circ \phi
$$

does not in general determine uniquely the derivations $d_{A}, d_{B}$. If $\phi$ is surjective then for a given $\left(\phi, \Phi, d_{A}\right)$ there exists at most one $d_{B}$ rendering commutative the diagram (1). The existence problem will follow from an additional assumption so called consistency conditions.

In fact we will be more specific. Assume that $\phi, \Phi$ are surjective homomorphisms and the diagram (1) commute. One has $B \cong A / \operatorname{ker} \phi$ and $N \cong M / \operatorname{ker} \Phi$ i.e. $d_{B}$ (if exists) is a factor calculus on factor algebra with values in factor bimodule. The differential $d_{A}$ is called a cover differential. In the next Section we shall study factor calculi and the corresponding consistency conditions with more details.

Lemma 1.2.2 ([2], p. 560). Let $V$ be a generating system for the algebra $A$. The diagram (1) is commutative if and only if it is commutative on elements from $V$. 
1.3. Universal problem for calculi. It is also very well known that for any algebra $A$ there exists an universal derivation $\delta_{A} \in \operatorname{der}_{\mathbb{k}}(A, \operatorname{ker} m)$ with values in a kernel of multiplication map $m$ and $\delta_{A}$ is defined by the formulae

$$
\delta_{A}=\mathrm{id}_{A} \otimes 1_{A}-1_{A} \otimes \mathrm{id}_{A} .
$$

with $1_{A}$ being a unit of $A$. It has the following universal property $[2,13]$ : for every $A$-bimodule $M$ and every derivation $d \in \operatorname{der}_{\mathbb{k}}(A, M)$, there exists one and only one $A$-bimodule homomorphism $\Phi_{d}: \operatorname{ker} m \rightarrow M$ such that $d=\Phi_{d} \circ \delta_{A}$. In other words an universal differential is a cover differential for any derivation $d$ of the algebra $A$ (one has $\phi_{d}=\mathrm{id}_{A}$ in this case). The existence is proved by setting

$$
\Phi_{d}\left(\sum_{i} x_{i} \otimes y_{i}\right)=\sum_{i} d x_{i} \cdot y_{i} \equiv-\sum_{i} x_{i} \cdot d y_{i}
$$

where, $\sum_{i} x_{i} \otimes y_{i} \in$ ker $m$. Thus we have a canonical $k$-module isomorphism

$$
\operatorname{hom}_{(A, A)}(\operatorname{ker} m, M) \stackrel{\delta_{A}}{\longleftrightarrow} \operatorname{der}_{\mathbb{k}}(A, M) .
$$

Remark 1 . Note that ker $m$ is generated (as a left or right $A$-module) by the image of $\delta_{A}$. Therefore if $M$ is also generated by differentials i.e. $M=d A . A=A . d A$ then $M$ is a factor bimodule of ker $m$ by some subbimodule. This condition was included by Woronowicz into the definition of calculi [18]. In this case $\Phi_{d}$ is surjective.

1.4. Calculi with partial derivatives. Assume that $k$ is a field. The algebra $A$ possesses a basis $\left\{1_{A}, e_{s}\right\}_{s \in S}$ (consisting finite or infinite number of elements) as a linear $\mathbb{k}$-space. One can easily observe (see e.g. [5]) that the set $\left\{e_{s} \otimes 1-1 \otimes e_{s}\right\}_{s \in S}$ is a set of free generators for the right (or left) $A$-module ker $m$. The condition that bimodule $M$ of one-forms admits free generators was assumed in the celebrated paper by Pusz and Woronowicz [14]. It was also a guiding line (although not assumed explicitly) in the paper by Wess and Zumino [17].

Let $d \in \operatorname{der}_{\mathbb{k}}(A, M)$ and assume that the bimodule $M$ as a right $A$-module is free. Let elements $\left\{\xi^{\gamma}\right\}_{\gamma \in \Gamma}$ be free generators of $M$ as a right module. One has in this case

$$
d a=\sum_{\gamma \in \Gamma} \xi^{\gamma} D_{\gamma} a
$$

where, the partial derivatives $D_{\gamma} \in \operatorname{hom}_{\mathbb{k}}(A, A)$ are uniquely defined. We call such calculus a calculus with partial derivatives. In particular, if $\left\{x^{\gamma}\right\}_{\gamma \in \Gamma}$ is a set of generators of the algebra $A$ such that $d x^{\gamma}=\xi^{\gamma}$ then the corresponding calculus is called coordinate calculus. Recently there is a great interest in non commutative differential calculi mainly with connection to quantum groups and covariant calculi on quantum hyperplains [9-12] and [14-18]. Many examples of coordinate calculi and calculi with partial derivatives have been studied in the literature (see e.g. $[1,5,6,9,15]$ ). More systematic approach and general formalism for coordinate calculi and calculi with partial derivatives over a field have been proposed $[3,4]$.

Let $W$ denote a free $\mathbb{k}$-module with basis $\left\{\xi^{\gamma}\right\}_{\gamma \in \Gamma} . W \otimes A$ has a canonical right $A$-module structure and with this structure it is isomorphic (but not canonically) to $M$ when considered as a right $A$-module. 
Let $V$ be a generating $k$-module for the algebra $A$. The aim of the present paper is to describe differential calculi on bimodules of the type $W \otimes A$ where, $V, W$ are some $l k$ modules. We do not assume in general that $V, W$ are free modules. In this way one obtains coordinate-free and basis-independent generalization of calculi with partial derivatives as well as a generalization to the case when $k$ is a commutative ring. This will be done in Section 3.

Our approach to non-commutative first-order differential calculi is traditionally a covariant one: it is done by means of differential one-forms. An extension to forms of higher degree (quantum de Rham complexes) can be found e.g. in [4, 10-12, 14, 17, 18]. A contravariant (i.e. by means of vector fields) approach will be proposed in [7].

2. Factor calculus on factor algebra with factor bimodule. Let now $A, B$ be two algebras, $M$ an $A$-bimodule and $N$ a $B$-bimodule. Notice that any homomorphism $\phi: A \rightarrow B$ induces an $A$-bimodule structure on $N$ (change of scalars) by

$$
x . n=\phi(x) . n, \quad n . x=n . \phi(x) .
$$

We denote this new $A$-bimodule structure on $N$ by $\phi^{*}(N)\left(\phi^{*}(N)=N\right.$ as sets $)$.

In the rest of this section $A, B$ are $\mathbb{l}$ algebras, $M$ is an $A$-bimodule and $\phi: A \rightarrow B$ is an surjective homomorphism. Recall that annihilator of a subbimodule $X \subseteq M$ in $A$ is a two sided ideal ann $X$ defined by

$$
\text { ann } X=\{a \in A: a . X=X . a=0\}
$$

2.1. Factor bimodule. In what follows we shall need the obvious criterion for a factor module $M / L$ to be an $A$-bimodule.

LEMma 2.1.1. Let $L$ be a $\mathbb{k}$-submodule of the A-bimodule $M$. The following are equivalent:

(i) $L$ is an A-subbimodule of $M$.

(ii) $M / L$ with the induced multiplication is an A-bimodule.

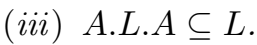

(iv) $A . L+L . A \subseteq L$.

One sees that for given $k$-submodule $L, \operatorname{lin}(A . L . A)$ is the smallest $A$-subbimodule of $M$ containing $L$.

Proposition 2.1.2. Let $I=\operatorname{ker} \phi$. Then $M=\phi^{*} N$ for some $B$-module $N$ if and only if $I \subseteq$ ann $M$.

Proof. For a case of right modules the proof can be found in ref. [13] p.23 .

In other words we get a condition for the $A$-bimodule $M$ to become a $B \cong A / I$ module. Below we will make no distinction between $A / I$-bimodules and $A$-bimodules $M$ if $I \subseteq$ ann $M$. The ideal ann $M$ is a maximal ideal possessing this property. A faithful bimodule $($ ann $=0)$ cannot be a bimodule over proper factor algebra in a way as describe above.

Proposition 2.1.3 Let $L$ be a subbimodule of $M$ and $I<A$ an ideal of $A$. Then the structure of $A$-bimodule on $M$ induces a structure of $A / I$-bimodule on $M / L$ if and only 
if

$$
I . M+M . I \subseteq L .
$$

Proof. From the previous Proposition we have to have $I \subseteq$ ann $M / L$, which is equivalent to $I .(M / L)=(M / L) \cdot I=0$. It gives the proof.

One sees that $\operatorname{lin}(I . M+M . I)$ is a subbimodule of $M$. It is the smallest subbimodule satisfying the statement above. We shall call the condition (5) compatibility condition between an ideal $I$ and a subbimodule $L$. Therefore, $\operatorname{lin}(I . M+M . I)$ is the smallest $I$-compatible subbimodule of $M$. If $I$ and $L$ are compatible then the factor module $M / L$ becomes a module over factor algebra $A / I$. In particular, if $I \subseteq$ ann $M$ i.e. $M . I=I . M=$ 0 , then any subbimodule $L$ is $I$-compatible. Observe that for a faithful bimodule $M$, $M / \operatorname{lin}(I . M+M . I)$ is in some sense a maximal factor bimodule over factor algebra $A / I$. On the other hand, if a subbimodule $L$ is fixed then there exists the largest $L$-compatible ideal $I(L)$ (the sum of all ideals satisfying (5)). The algebra $A / I(L)$ is in some sense a minimal algebra compatible with the subbimodule $L$.

2.2. Factor calculus on factor algebra with factor bimodules. Combining Proposition 1.2.1 with the results above we have

Theorem 2.2.1. (Factor calculi) Let $I<A$ be an ideal in $A, L$ be a subbimodule of an $A$-bimodule $M$ and $d \in \operatorname{der}_{\mathbb{k}}(A, M)$. In order to exist a unique factor calculus $\tilde{d} \in \operatorname{der}_{k}(A / I, M / L)$ such that

$$
\Pi \circ d=\tilde{d} \circ \pi
$$

where, $\pi: A \rightarrow A / I$ and $\Pi: M \rightarrow M / L$ are canonical surjections, it is sufficient and necessary that

$$
d I+I . M+M . I \subseteq L .
$$

Proof. We know that $M . I+I . M \subseteq L$ guarantees existence of an $A / I$ bimodule structure on $M / L$. One also knows that $\tilde{d}$ if exists is unique. Moreover $\tilde{d}(x+I)=d x+L$ is well defined if and only if $d I \subseteq L$. This leads to commutativity condition (6). To complete the proof one has to apply the Proposition 1.2.1 .

Remark 2. Notice that in the case $I=0$ i.e. $\pi=\mathrm{id}_{A}$ there is no any condition for existence of factor calculi on a factor bimodule $M / L$.

When $L$ is a $\mathbb{I} k$-module then the condition (7) must be replaced by (c.f. Lemma 2.1.1)

$$
d I+M . I+I . M+A . L . A \subseteq L .
$$

The conditions of the type $(7,8)$ for existence a factor derivation are usually in the literature called consistency conditions for a factor calculi. They include a compatibility condition between an ideal $I$ and $A$-subbimodule ( $k$-submodule) $L$. Notice that in this case the cover differential $d$ is not in general, uniquely determined by the diagram (1).

Again one can consider some special situations. Firstly, note that if $I \subseteq$ ker $d$ then (5) is the only condition ensuring the existence of factor calculus. Secondly, for a given $I$, $\operatorname{lin}(d I+M . I+I . M)$ is the smallest subbimodule admitting a factor calculus. Finally if $L$ 
is fixed then there exists a maximal ideal $I(d, L)$ and the corresponding optimal algebra with a factor calculus. Notion of optimal algebra has been introduced in [3] (c.f. [4-6]).

\section{Calculi with right-universal bimodules}

3.1. Right-universal modules. Let $A$ be an algebra and $W$ a $\mathbb{k}$-module. Consider the $I k$-module $W \otimes A$. It has a canonical right $A$-module structure $(w \otimes x) \cdot y=w \otimes x y$ and with this structure $W$ generate $W \otimes A$. Observe that in the case $k$ is a field $W \otimes A$ is a free right module and $W$ plays the role of space spanned by its free generators. Universal bimodules of the form $A \otimes W \otimes A$ has been considered in [8].

Notice the following universal property of $W \otimes A$ : for each $l k$-linear mapping $\Phi$ from $W$ into a right $A$-module $M$ there exists a unique $A$-right module homomorphism $\Phi^{u}: W \otimes A \rightarrow M$ (called an universal lift of $\left.\Phi\right)$, such that

$$
\Phi^{u} \circ i=\Phi
$$

where, $i(w)=w \otimes 1_{A}$ is a canonical mapping $i: W \rightarrow W \otimes A$. $\Phi^{u}$ is defined by setting $\Phi^{u}(w \otimes x)=\Phi(w) . x$ and its image is the right submodule generated by $W$. Therefore, $\Phi^{u}$ is surjective if and only if $\Phi(W)$ generates $M$. In other words each right $A$-module generated by $W$ is a factor module of $W \otimes A$. More exactly we have

Lemma 3.1.1. Let $W$ be a $\mathbb{k}$-submodule of a right $A$-module $M . W$ generates $M$ if and only if

$$
M \cong(W \otimes A) / \operatorname{ker} i d_{W}^{u}
$$

where, $i d_{W}^{u}$ is an universal lift of the identity map $i d_{W}: W \rightarrow W \subseteq M$.

If $\operatorname{kerid}_{W}^{u}=0$ then $W$ is said to be a space of universal generators of $M$. In this case $M \cong W \otimes A$ and is said to be a right-universal module generated by $W$. $W$ is said to be a $k$-module of universal generators of $M$.

Not e. We do not assume that the $\mathbb{l}$-module $W$ possesses free generators, i.e. that $W$ is a free $l k$-module. Therefore, $W \otimes A$ is not a free right $A$-module, in general. However, starting from the Subsection 3.4 we shall assume projectivity of $W$.

The map $u$ which establishes the correspondence between a $l k$-linear maps from $W$ into $M$ and an $A$-linear maps from $W \otimes A$ into $M$

$$
\operatorname{hom}(W, M) \longleftrightarrow \operatorname{hom}_{A}(W \otimes A, M)
$$

is $l k$-linear and bijective.

3.2. Left A-module structure. Since for calculi we need bimodule structure rather then right module we investigate now how to determine a left module structure on $W \otimes A$ in such a way that $W \otimes A$ becomes a bimodule.

Recall that a left $A$-module structure on a $l k$-module $M$ is given by an arbitrary element of $\operatorname{alg}(A$, End $M)$. Due to a canonical isomorphism of $l k$-modules (see [2] p. 267)

$$
\operatorname{hom}(A, \text { End } M) \cong \operatorname{hom}(A \otimes M, M)
$$

one has $\operatorname{alg}(A$, End $M) \subset \operatorname{hom}(A \otimes M, M)$. 
When $M$ is a right $A$-module then we are interested in such left module structures which convert $M$ into an $A$-bimodule. The set of such structures is now identical with $\operatorname{alg}\left(A\right.$, End $\left._{A} M\right) \subset \operatorname{hom}_{A}(A \otimes M, M)$.

Let $V, W$ be two $\mathbb{k}$-modules. In this note by a twisting map or simply a twist we shall call an arbitrary $\mathbb{k}$-linear map from $V \otimes W$ into its twist $W \otimes V$.

Proceeding to the case of a right-universal $A$-module $W \otimes A$ one sees by the universal mapping property (10) that $\operatorname{hom}_{A}(A \otimes W \otimes A, W \otimes A)$ is isomorphic to $\operatorname{hom}(A \otimes W, W \otimes A)$. Consider arbitrary twisting map $b: A \otimes W \rightarrow W \otimes A$. We will be interested to know when its universal lift $b^{u}: A \otimes W \otimes A \rightarrow W \otimes A$ determines an $A$-bimodule structure on the right-universal $A$-module $W \otimes A$. Thus

Lemma 3.2.1. Let $A$ be an algebra and $W$ a $k$-module. A twisting map $b: A \otimes W \rightarrow$ $W \otimes A$ defines a bimodule structure on $W \otimes A$ if and only if the universal lift $b^{u}$ is an algebra homomorphism i.e. $b^{u} \in \operatorname{alg}\left(A, \operatorname{End}_{A}(W \otimes A)\right)$.

P r o of. One should remember that in this case $\operatorname{alg}\left(A, \operatorname{End}_{A}(W \otimes A)\right) \subset \operatorname{hom}_{A}(A \otimes$ $W \otimes A, W \otimes A)$ by a canonical isomorphism mentioned above.

Proposition 3.2.2. There is a one to one correspondence between left A-module structures transforming $W \otimes A$ into bimodule and twisting maps $b: A \otimes W \rightarrow W \otimes A$ satisfying:

(i) $b\left(1_{A} \otimes w\right)=w \otimes 1_{A}$

(ii) $b \circ\left(m \otimes i d_{W}\right)=\left(i d_{W} \otimes m\right) \circ\left(b \otimes i d_{A}\right) \circ\left(i d_{A} \otimes b\right)$ on $A \otimes A \otimes W$.

Pr o of. $W \otimes A$ already has a right $A$-module structure. $b$ determines a left multiplication by $x .(w \otimes y)=b(x \otimes w) . y \equiv b^{u}(x \otimes w \otimes y)$. Bimodule axioms follow easily from the postulates.

Twistings satisfying the conditions (i) and (ii) above will be called bimodule commutation rules and the corresponding $A$-bimodule structure will be denoted by $W_{b} \otimes A$.

Lemma 3.2.3. Let $M$ be an A-bimodule. Assume that the $\mathbb{R}$-submodule $W$ universally generates $M$ as a right $A$-module. Then there exists a unique bimodule commutation rule $b: A \otimes W \rightarrow W \otimes A$ such that $M \cong W_{b} \otimes A$.

Proof. Let $\Phi: W \otimes A \rightarrow M$ denotes an isomorphism of right modules. Define $b(x \otimes w)=\Phi^{-1}(x . w)$. The proof is done.

$W_{b} \otimes A$ is said to be a right-universal bimodule generated by $W$ with a left module structure defined by the bimodule commutation rule $b$.

Our next task is to investigate calculi on factor modules of the type $W \otimes(A / I) \cong$ $(W \otimes A) /(W \otimes I)$, where $I$ is an ideal of $A$.

Note. To be precise one has to distinguish between $l k$-module $W \otimes I$ and its image $\operatorname{Im}(W \otimes I) \subset W \otimes A$ under the canonical map $\operatorname{id}_{W} \otimes i$ where, $i: I \rightarrow A$ is an injection (see [2] p. 252). Hence, $W \otimes(A / I) \cong(W \otimes A) / \operatorname{Im}(W \otimes I)$ in general. For a projective $l k$-module $W$ it is possible to identify $W \otimes I$ with its image.

With this notation one has

Proposition 3.2.4. Let $I<A$ be an ideal of $A$ and $W$ be a projective $\mathbb{l}$-module. The bimodule commutation rule $b: A \otimes W \rightarrow W \otimes A$ factorize to the bimodule commutation 
rule $\tilde{b}:(A / I) \otimes W \rightarrow W \otimes(A / I)$ if and only if

$$
b(I \otimes W) \subseteq W \otimes I .
$$

If in addition $d: A \rightarrow W_{b} \otimes A$ is a calculus then the condition

$$
d I \subseteq W \otimes I
$$

is sufficient and necessary for existence of a factor calculus $\tilde{d}: A / I \rightarrow W_{\tilde{b}} \otimes(A / I)$.

P r o of. First consider factor bimodule structure. Take $L=W \otimes I$. We have to prove that $L$ is an $A$ - subbimodule of $W_{b} \otimes A$ (Lemma 2.1.1) which satisfies the condition (5) of Proposition 2.1.2 . It is clear that $L . A \subseteq L$ and $M . I \subseteq L$. It means that the factor Ik-module $W \otimes(A / I) \cong(W \otimes A) / L$ has a right $A / I$-module structure and $W$ universally generates it as a right $A / I$ module. Next calculate $A . L=b^{u}(A \otimes W \otimes I)=b(A \otimes W) . I \subset$ $(W \otimes A) . I \subseteq L$. To see $I . M \subseteq L$ one has to use (12a). Indeed $I . M=b^{u}(I \otimes W \otimes A)=$ $b(I \otimes W) . A \subseteq(W \otimes I) . A \subseteq L$. Therefore, we have a bimodule structure on a factor module $W \otimes(A / I)$. Then by Lemma 3.2 .3 it is isomorphic to $W_{\tilde{b}} \otimes(A / I)$ with some commutation rule $\tilde{b}:(A / I) \otimes W \rightarrow W \otimes(A / I)$. In this case

$$
\tilde{b}((x+I) \otimes w)=b(x \otimes w)+L .
$$

Now the last statement is a simple consequence of Theorem 2.2.1 . The proof is finished.

The condition (12a) will be called a compatibility condition between an ideal $I$ and a bimodule commutation rule $b$. The conditions (12a) and (12b) form together consistency conditions for the existence of a factor calculus with values in right-universal bimodules.

Rem ark 3. Notice that in the conditions (12a), (12b) both sides depend on the ideal $I$. Therefore the existence of an optimal algebra is not in general an obvious question $[3-6]$.

There exists a canonical $k$-module homomorphism

$$
\lambda: \text { End }(W) \otimes A \longrightarrow \operatorname{hom}(W, W \otimes A)
$$

which in general is neither surjective nor injective ([2] p. 275 formulae (22)). It induces a homomorphism $\rho=\operatorname{hom}\left(\operatorname{id}_{A}, \lambda\right)([2]$ p. 229 and 267):

$$
\rho: \operatorname{hom}(A, \operatorname{End}(W) \otimes A) \longrightarrow \operatorname{hom}(A, \operatorname{hom}(W, W \otimes A)) \cong \operatorname{hom}(A \otimes W, W \otimes A)
$$

assigning to each $l k$-linear map $b: A \rightarrow$ End $(W) \otimes A$ a twist $b^{\rho}: A \otimes W \rightarrow W \otimes A$ by the formulae

$$
b^{\rho}(x \otimes w)=b(x)(w) \in W \otimes A
$$

where, $b^{\rho} \equiv \rho(b)$. It is not difficult to check that the image $\rho(\operatorname{alg}(A$, End $(W) \otimes A)) \subset$ $\operatorname{hom}(A \otimes W, W \otimes A)$ provides bimodule commutation rules. Therefore, the compatibility condition (11) can be replaced in this case by the following condition

$$
b^{\rho}(I) \subseteq \operatorname{End}(W) \otimes I .
$$

$\lambda$ is bijective (it is so if e.g. $W$ is finitely generated and projective $\mathbb{l}$-module [2] p. $274)$ if and only if $\rho$ is bijective ([2] p. 229). This approach is more convenient for the case when $k$ is a field (c.f. $[3-6])$. 
3.3. Calculi on tensor algebra. Let $V$ be a $l k$-module and $T V$ denotes its tensor algebra. (We neglect a graded algebra structure of $T V$ at this stage). It is a free $\mathbb{k}$-algebra generated by $V$ since it possesses the following universal property: for each linear map $g$ from $V$ into algebra $A$ there exists a unique algebra homomorphism $g_{u}: T V \rightarrow A$ (called again an universal lift of $g$ ), such that

$$
g_{u} \circ j=g
$$

where, $j$ denotes a canonical inclusion of $V$ into $T V$. The image of $g_{u}$ is a subalgebra generated by $g(V)$. In particular, if $V \subseteq A$ generates $A$ then $A \cong T V / I$ where, $I=\operatorname{ker} g_{u}$ is an ideal of relations in $A$. In other words any algebra generated by $V$ is a quotient of $T V$. Again the correspondence between $k$-linear maps from $V$ into $A$ and algebra maps from $T V$ into $A$ i.e. between

$$
\operatorname{hom}(V, A) \longleftrightarrow \operatorname{alg}(T V, A)
$$

is an isomorphism of $\mathbb{l}$-modules.

Not e. Let $I<T V$ be a two-sided ideal. It appears that in general the factor algebra $T V / I$ does not contain $V$ as a $l k$-submodule. Some of the results below are valid in the general situation $T V / I$. Some other, require an additional assumption that $V \subseteq T V / I$. In this case we shall speak that algebra is generated by its submodule $V$. Here $V$ need not to be a free $\mathbb{l} k$-module. Therefore, $T V$ is not a free algebra in general.

Recall that $V$ is a projective $\mathbb{k}$-module if and only if for every surjective ( $k$-linear) map $\Pi: P \rightarrow Q$ and every map $f: V \rightarrow Q$ there exists a map $\hat{f}: V \rightarrow P$ such that $\hat{f} \circ \Pi=f$. It is said that $f$ can be lifted to a map of $V$ into $P$ or that $\hat{f}$ covers $f$ subject to the epimorphism $\Pi$. Of course, $\hat{f}$ exists but is not unique in general. Tensor product of projective modules (over commutative ring) is again projective.

From now on, up to the end of this note we shall assume that $V$ and $W$ are projective $I k$-modules. In a case of $V$-universal algebra $T V$ one has (c.f. [4])

Proposition 3.3.1 Any (linear) map $b_{0}: V \otimes W \rightarrow W \otimes T V$ determines uniquely a bimodule structure on $W \otimes T V$. Conversely, any bimodule commutation rule on $W \otimes T V$ can be obtained in this way.

P r o of. We shall make use of canonical isomorphisms listed above. First by the canonical lift (10) we get an element $\left(b_{0}\right)^{u} \in \operatorname{hom}_{T V}(V \otimes W \otimes T V, W \otimes T V)$ which is a $T V$-right module map. Next, by a canonical isomorphism (11) $\left(b_{0}\right)^{u} \in \operatorname{hom}\left(V, \operatorname{End}_{T V}(W \otimes T V)\right)$. Finally, a canonical lift (14) gives an algebra map $\left(\left(b_{0}\right)^{u}\right)_{u} \in \operatorname{alg}\left(T V, \operatorname{End}_{T V}(W \otimes T V)\right)$. This is a required left $T V$-module structure on $W \otimes T V$. By Lemma 3.2.1 it leads to the bimodule commutation rule $b: T V \otimes W \rightarrow W \otimes T V$ such that $b^{u}=\left(\left(b_{0}\right)^{u}\right)_{u}$. It finishes the proof.

R e mark 4. Observe that since $T V=\oplus_{n \geq 0} V^{\otimes n}$ is a graded algebra then $W \otimes T V \equiv$ $\oplus_{n \geq 0} W \otimes V^{\otimes n}$ is a graded right module. Therefore, $b$ preserve gradation imply that $W_{b} \otimes T V$ is a graded bimodule. If in addition $I$ is a graded ideal then factor bimodule is again graded. Replace word 'graded' by 'filtered'. The same is true. 
Proposition 3.3.2. (Calculus on tensor algebra) For each two Ik-linear maps $d_{0}$ : $V \rightarrow W \otimes T V$ and $b_{0}: V \otimes W \rightarrow W \otimes T V$ there exists exactly one calculus $d$ on $T V$ with values in $W_{b} \otimes T V$ such that $\left.d\right|_{V}=d_{0}$ and $\left.b\right|_{V \otimes W}=b_{0}$. Moreover in the graded case i.e. $b_{0}: V \otimes W \rightarrow W \otimes V$ and $d_{0}: V \rightarrow W \otimes 1_{A}$, the derivation $d$ is a graded map of degree -1 .

Pr o of. (c.f. also $[3,4])$. Let $M$ be a $T V$-bimodule and $d_{0}: T V \rightarrow M$ a $\mathbb{k}$-linear map. One has to define an $M$-valued $l k$-derivation $d$ on $T V . V$ generates $T V$. By $\mathbb{k}$-linearity it is enough to define $d$ on monomials $v_{1} \otimes v_{2} \otimes \ldots \otimes v_{k} \in T V$ where, $v_{1}, v_{2}, \ldots, v_{k} \in V$. Do it by induction.

(i) $d v=d_{0}(v)$ for $v \in V$.

(ii) If $x=v \otimes x_{1}$ is a word of length $k, v \in V$ and $x_{1}$ is a word of length $(k-1)$ then define

$$
d x=(d v) \cdot x_{1}+v \cdot d x_{1}=\left(d_{0} v\right) \cdot x_{1}+v \cdot d x_{1} .
$$

Now, we have to prove that $d$ satisfies the Lebniz rule. Let $x, y$ be two monomials and $x=v \otimes x_{1}$ as before. Again by inductive assumption if $d\left(x_{1} \otimes y\right)=\left(d x_{1}\right) \cdot y+x_{1} \cdot d y$ then

$$
\begin{gathered}
d(x \otimes y)=d\left(v \otimes\left(x_{1} \otimes y\right)\right)=\left(d_{0} v\right) \cdot\left(x_{1} \otimes y\right)+v \cdot d\left(x_{1} \otimes y\right)= \\
=\left(\left(d_{0} v\right) \cdot x_{1}\right) \cdot y+v \cdot\left(d x_{1} \cdot y+x_{1} \cdot d y\right)=d x \cdot y+x \cdot d y
\end{gathered}
$$

since $v \cdot\left(x_{1} \cdot d y\right)=\left(v \otimes x_{1}\right) \cdot d y=x . d y$. Thus we have prooved that any $\mathbb{l}$-linear map $d_{0}: V \rightarrow M$ uniquely extends to a $l k$-derivation $d: T V \rightarrow M$. Obviously, any calculus on $T V$ can be obtained in this way.

Next, apply above result to the case $M=W_{b} \otimes R V$ where, $b: T V \otimes W \rightarrow W \otimes T V$ denotes the commutation rule constructed out of $b_{0}$ in the proof of Proposition 3.3.1 .

Finally, in the homogeneous case, $d_{0} v \in \mathbb{R}$ and $b_{0}: V \otimes W \rightarrow W \otimes V$. In this case $b$ preserve the grading (which is a length of monomials). Therefore, by inductive formulae $d$ decreases a degree by one. The proof is done.

In other words calculus on tensor algebra with values in right universal bimodule is completely determined by the initial data: $b_{0}$ and $d_{0}$.

Proposition 3.3.3. Let calculus $d: T V \rightarrow W_{b} \otimes T V$ is given. The condition

$$
d I+b(I \otimes W) \subseteq W \otimes I .
$$

is a sufficient and necessary for the existence (a unique) factor calculus $\tilde{d}: T V / I \rightarrow$ $W_{\tilde{b}} \otimes(T V / I)$ where, $\tilde{b}$ denotes a factor commutation rule.

Pr o of. It is a simple consequence of Proposition 3.2.4.

Rem ark 5. It is clear that the condition (15) can be equivalently replaced by two conditions

$$
\begin{gathered}
d I \subseteq W \otimes I \\
b(I \otimes W) \subseteq W \otimes I \quad\left(\Longleftrightarrow b^{\rho}(I) \subseteq \operatorname{End}(W) \otimes I\right)
\end{gathered}
$$

(Formulae in brackets relates to the case governed by (13)). For the quadratic algebras this type of conditions, when written in the operator form (see below) have been introduced in [17]. Therefore, they are often called Wess-Zumino linear and quadratic consistency 
conditions. In some cases it is possible to express the consistency conditions in terms of generating $l k$-module for the ideal $I$. It will be studied in Subsection 3.5.

One sees from the above considerations that factor calculi of calculi on universal algebra form an important class of calculi. This is so due to the following two facts. Firstly, we are able to describe all possible calculi on universal algebra with values on right-universal bimodule. Secondly, the consistency conditions (15a, b) are convenient and more easy to handle. In the next point we answer the question: under which circumstances all calculi on the algebra $A$ can be obtained in this way.

3.4. Calculi with right-universal bimodule-structure theorem. Joining the universal properties of $W \otimes A$ with that of $T V$ one has: Let $A$ be an algebra generated by $\mathbb{k}$ submodule $V \subseteq A$ and $M$ be a right-universal $A$-bimodule universally generated by its submodule $W \subseteq M$. Then $A \cong T V / I$ and $M \cong W_{b} \otimes(T V / I)$ for some bimodule commutation rule $b$. We show our main result that each calculus on $T V / I$ with values in right-universal bimodule $W_{b} \otimes A$ is a factor calculus of some cover calculus $\hat{d}$ on tensor algebra $T V$ subject to the cover bimodule commutation rule $\hat{b}: T V \otimes W \rightarrow W \otimes T V$. More exactly one has

THEOREM 3.4.1. (Main result. Cover calculus.) With the notation as above:

Let $d: T V / I \rightarrow W_{b} \otimes(T V / I)$ be a differential calculus. There exist (not unique in general) initial data $\hat{b}_{0}: V \otimes W \rightarrow W \otimes T V$ and $\hat{d}_{0}: V \rightarrow W \otimes T V$ such that the corresponding calculus $\hat{d}$ on $T V$ factorize to $d$. Thus the diagram

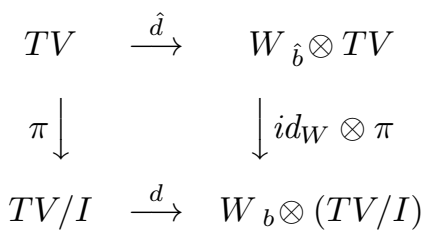

is commutative where, $\pi: T V \rightarrow T V / I$ is a canonical surjection. Moreover, the following consistency condition is satisfied

$$
\hat{d} I+\hat{b}(I \otimes W) \subseteq W \otimes I .
$$

P r o of. Since $\pi$ and $\operatorname{id}_{W} \otimes \pi$ are in particular surjective $l k$-linear maps one can apply projectivity of $V$ and $V \otimes W$. Set $b_{0}=\left.b\right|_{V \otimes W}: V \otimes W \rightarrow W \otimes A$ and $d_{0}=\left.d\right|_{V}: V \rightarrow$ $W \otimes A$. Let $\widehat{b_{0}}: V \otimes W \rightarrow W \otimes T V$ and $\widehat{d_{0}}: V \rightarrow W \otimes T V$ be some their (not unique) lifts. By Proposition 3.3.2 let $\hat{d}$ denotes the corresponding (unique) calculus on $T V$. To show commutativity of the diagram ( it is enough to check it on generating space $V$ (Lemma 1.2.2). We leave it to the reader (c.f. $[3,4]$ ). The consistency condition (16) follows easily from Proposition 3.3.3.

Remark 6. So called coordinate calculus [3-6] corresponds to the case $W=V$ be a vector space and $d_{0}=\mathrm{id}_{V}$.

Theorem shows the way to construct and classify all calculi with right-universal bimodules if one has been given the $k$-projective modules of generators of an algebra and a bimodule. In order to do this we have to follow one of two main strategies: 
- fix the ideal $I$ in the tensor algebra and therefore an algebra $A$ and its presentation $A \equiv T V / I$. Look for all initial data $d_{0}, b_{0}$ such that the corresponding calculus $d$ on $T V$ satisfies the consistency conditions $(15 \mathrm{a}, \mathrm{b})$;

- choose the initial data. Look for all consistent ideals $I$.

3.5. Applications. Calculi on quadratic algebras. Let $V$ be a $l k$-module and $K \subseteq V^{\otimes 2}$ be a submodule. Denote by $\langle K\rangle$ an ideal generated in $T V$ by $K$. The factor algebra $T V /\langle K\rangle$ is said to be an algebra with quadratic relations (quadratic algebra in short). It is not difficult to see that the ideal $\langle K\rangle$ is a graded ideal $\langle K\rangle=\oplus_{n \geq 2} K_{n}$ with the homogeneous components satisfying the recurrent relations

$$
K_{n+1}=V \otimes K_{n}+K_{n} \otimes V .
$$

where, $K_{2} \equiv K$. Let $W$ be another $l k$-module. Assume we are given the derivation $d: T V \rightarrow W \otimes T V$ with homogeneous initial data $b_{0}: V \otimes W \rightarrow W \otimes V$ and $d_{0}: V \rightarrow W$. In this case the derivation $d$ decreases the degree. Therefore, the linear consistency condition (15a) takes the very simple form

$$
d K=0 .
$$

Taking into account the Leibniz rule and homogeneity of $d$ we get

$$
\left.\left[d_{0} \otimes \mathrm{id}_{V}+b_{0} \circ\left(\mathrm{id}_{V} \otimes d_{0}\right)\right]\right|_{K}=0 .
$$

Also quadratic consistency condition (15b) simplify to

$$
b(K \otimes W) \subseteq W \otimes K\left(\Longleftrightarrow b^{\rho}(K) \subseteq \operatorname{End}(W) \otimes K\right)
$$

where, $b: T V \otimes W \rightarrow W \otimes T V$ is an associated bimodule commutation rule. To see this one has to apply algebra homomorphism $b^{\rho}$ to formulae (17). It appears that (20) can be further simplify to

$$
\left(b_{0} \otimes \operatorname{id}_{V}\right) \circ\left(\operatorname{id}_{V} \otimes b_{0}\right)(K \otimes W) \subseteq W \otimes K
$$

if we use Proposition 3.2.1.

Our aim in this Section is to find out an operator (matrix) form of consistency condition $(19,21)$. Thus one has (c.f. $[5,12])$

Proposition 3.5.1. Let $c \in \operatorname{End}\left(V^{\otimes 2}\right)$ be a twist with the property that $\operatorname{Im} c \equiv$ $c(V \otimes V)=K$. Then the consistency condition (19, 21) for the homogeneous calculus on the quadratic algebra $T V /<\operatorname{Im} c>$ are equivalent to the following conditions

$$
\begin{gathered}
{\left[d_{0} \otimes i d_{V}+b_{0} \circ\left(i d_{V} \otimes d_{0}\right)\right] \circ c=0} \\
\left(b_{0} \otimes i d_{V}\right) \circ\left(i d_{V} \otimes b_{0}\right) \circ\left(c \otimes i d_{W}\right)=\left(i d_{W} \otimes c\right) \circ a
\end{gathered}
$$

for some twist $a: V^{\otimes 2} \otimes W \rightarrow W \otimes V^{\otimes 2}$.

ExAmple. Let $b_{0}: V \otimes W \rightarrow W \otimes V$ be simply a switch i.e. $b_{0}(v \otimes w)=w \otimes v$. Then for any ideal $I<T V$ the quadratic consistency condition (15b) is automatically satisfied. It follows that for any $\mathbb{l}$-algebra $A \equiv T V / I$ generated by $V$, one has $b(x \otimes w)=w \otimes x$ and $x .(w \otimes y) . z=w \otimes(x y z)$ in $W_{b} \otimes A$. The linear consistency condition (15a) is the 
only one to be fulfilled for the existence of factor calculus on $A$. In the quadratic algebra case with $d_{0}: V \rightarrow W$ (Proposition 3.5.1) (22) reads

$$
\left(d_{0} \otimes \mathrm{id}_{V}\right) \circ c=0
$$

i.e. $\operatorname{Im} c \subset \operatorname{ker}\left(d_{0} \otimes \mathrm{id}_{V}\right)$. Therefore, $T V /<\operatorname{ker}\left(d_{0} \otimes \mathrm{id}_{V}\right)>$ is an optimal algebra for these bimodule commutation rules.

Rem ark 7. Very peculiar solutions of the quadratic consistency condition (23) are given by solutions of the braid equation (quantum Yang-Baxter eq. ) vis.

$$
\left(b_{0} \otimes \mathrm{id}_{V}\right) \circ\left(\mathrm{id}_{V} \otimes b_{0}\right) \circ\left(c \otimes \mathrm{id}_{W}\right)=\left(\mathrm{id}_{W} \otimes c\right) \circ\left(b_{0} \otimes \mathrm{id}_{V}\right) \circ\left(\mathrm{id}_{V} \otimes b_{0}\right)
$$

on $V^{\otimes 2} \otimes W$. (See [5] for more examples and comments.)

\section{References}

[1] H. C. Baehr, A. Dimakis and F. Müller-Hoissen, Differential Calculi on Commutative Algebras, J. Phys. A: Math. Gen. 28 (1995), 3197-3222, hep-th/9412069.

[2] N. Bourbaki, Elements of mathematics.Algebra I.Chapters 1-3, Springer-Verlag, Berlin, 1989.

[3] A. Borowiec, V. K. Kharchenko and Z. Oziewicz, On free differentials on associative algebra, in : Non-Associative Algebra and Its Applications, S. González (ed.), Kluwer Academic Publishers, Dordrecht 1994, ISBN 0-7923-3117-6, Mathematics and its Applications, vol. 303, 46-53, (hep-th/9312023).

[4] A. Borowiec and V. K. Kharchenko, Algebraic approach to calculi with partial derivatives, Siberian Advances in Mathematics 5, 2 (1995), 10-37.

[5] A. Borowiec and V. K. Kharchenko, Coordinate calculi on associative algebras, in : Quantum Group, Formalism and Applications, J. Lukierski, Z. Popowicz and J. Sobczyk (ed.), Polish Sci. Publ. PWN Ltd., Warszawa, 1995. ISBN 83-01-11770-2, 231-241, q-alg/9501018.

[6] A. Borowiec and V. K. Kharchenko, First order optimum calculi, Bull. Soc. Sci. Lett. Łódź v. 45, Ser. Recher. Deform. XIX, (1995), 75-88, q-alg/9501024.

[7] A. Borowiec, Cartan Pairs, Czech. J. Phys. 46, 12 (1996), 1197 (q-alg/9609011).

[8] J. Cuntz and D. Quillen, Algebra Extension and Nonsingularity, J. Amer. Math. Soc. 8, 2 (1995), p. 251-289.

[9] A. Dimakis, F. Müller-Hoissen and T. Striker, Non-commutative differential calculus and lattice gauge theory, J. Phys. A: Math. Gen. 26 (1993), 1927-1949.

[10] G. Maltsiniotis, Le Langage des Espaces et des Groupes Quantiques, Commun. Math. Phys. 151 (1993), 275-302.

[11] Yu. I. Manin, Notes on quantum groups and quantum de Rham complexes., Preprint, MPI/91-60 (1991).

[12] E. E. Mukhin, Yang-Baxter operators and noncommutative de Rham complexes, Russian Acad. Sci. Izv. Math. 58, 2 (1994), 108-131 (in Russian).

[13] R. S. Pierce, Associative algebras, Graduate Texts in Mathematics \# 88, SpringerVerlag, New York, 1982.

[14] W. Pusz and S. Woronowicz, Twisted second quantization, Reports on Mathematical Physics 27, 2 (1989), 231-257. 
[15] W. Pusz, Twisted canonical anticommutation relations, Reports on Mathematical Phys. 27, 3 (1989), 349-360.

[16] K. Schmüdgen and A. Schüler, Classification of bicovariant calculi on quantum spaces and quantum groups, C. R. Acad. Sci. Paris 316 (1993), 1155-1160.

[17] J. Wess and B. Zumino, Covariant differential calculus on the quantum hyperplane, Nuclear Physics 18 B (1990), 303-312, Proc. Suppl. Volume in honor of R. Stora.

[18] S. L. Woronowicz, Differential calculus on compact matrix pseudogroups (quantum groups), Commun. Math. Phys. 122 (1989), 125-170. 